\title{
Wild life surveillance on Echinococcus multilocularis in Sweden
}

\author{
Birgitta Andersson*, Bodil Christensson, Susanne Johansson, Eva Osterman Lind, Göran Zakrisson \\ From Parasite infections of domestic animals in the Nordic countries - emerging threats and challenges. \\ The 22nd Symposium of the Nordic Committee for Veterinary Scientific Cooperation (NKVet) \\ Helsinki, Finland. 7-9 September 2008
}

\section{Background}

Echinococcus multilocularis is a tapeworm whose adult stages parasitize the intestine of canids such as foxes and wolves. Also domestic dogs and cats can act as definitive hosts. The sylvatic life cycle includes small rodents as intermediate hosts but humans may become accidentally infected by ingestion of eggs. Sweden, Finland, UK, Ireland, and Malta are considered to be free of this parasite and therefore have maintained their national rules as regards deworming of pets at movement into the countries. According to the EC regulation, these national rules can be applied during a transitional period to 2010 .

In order to confirm the absence of E. multilocularis in Sweden, monitoring of foxes is being carried out continuously. These investigations are financed by the Swedish government.

\section{Materials and methods}

In 2007, 245 red foxes were shot and sent to SVA by local hunters in different parts of Sweden. To kill potential tapeworm eggs, the carcasses were placed in $-80^{\circ} \mathrm{C}$ for at least one week before sampling. Faecal samples were then collected from the rectum and sent to Switzerland for testing by coproantigen ELISA (Deplazes et al., 1999). Forty-eight foxes that were positive in the ELISA and additional 28 randomly selected individuals were also examined by a sedimentation technique according to the OIE guidelines.

\section{Results and discussion}

Forty-eight foxes out of 245 were positive for Echinococcus sp. by the coproantigen ELISA. With the sedimentation technique however, Echinococcus sp was not detected in any of the examined animals, including those who had been positive in the ELISA. One possible explanation for obtaining false positive ELISA results was that some kind of cross-reaction had taken place. The majority of the foxes were infected with other parasites, for example Taenia sp, Mesocestoides sp, Alaria alata, Toxocara canis, Toxascaris leonina.

\section{Conclusions}

There is a need for good screening methods with high sensitivities and specificities. The results obtained by the sedimentation technique indicate that Sweden was still free from the fox tapeworm in 2007.

Published: 13 October 2010

\section{Reference}

1. Deplazes P, Alther P, Tanner I, Thompson RCA, Eckert J: Echinococcus multilocularis coproantigen detection by enzyme-linked immunosorbent assay in fox, dog and cat populations. J. Parasitol 1999, 85:115-121.

doi:10.1186/1751-0147-52-S1-S10

Cite this article as: Andersson et al:: Wild life surveillance on

Echinococcus multilocularis in Sweden. Acta Veterinaria Scandinavica 2010 52(Suppl 1):S10.
National Veterinary Institute, Department of Virology, Immunobiology and Parasitology, Section for Parasitological Diagnostics; SE-751 89 Uppsala, Sweden 